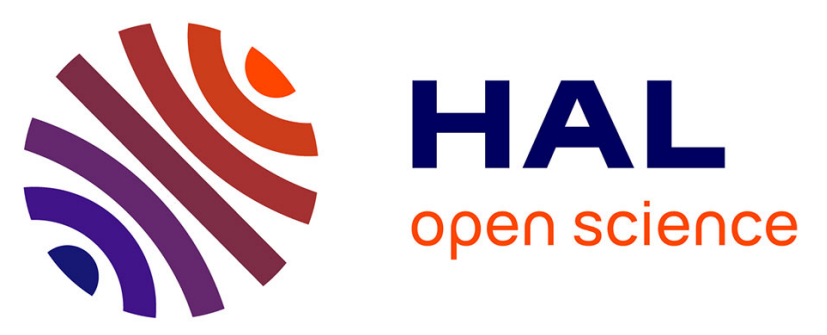

\title{
Surface decoration of catanionic vesicles with superparamagnetic iron oxide nanoparticles: a model system for triggered release under moderate temperature conditions
}

G. Bealle, L. Lartigue, C. Wilhelm, J. Ravaux, F. Gazeau, Renaud Podor, David Carriere, C. Menager

\section{To cite this version:}

G. Bealle, L. Lartigue, C. Wilhelm, J. Ravaux, F. Gazeau, et al.. Surface decoration of catanionic vesicles with superparamagnetic iron oxide nanoparticles: a model system for triggered release under moderate temperature conditions. Physical Chemistry Chemical Physics, 2014, 16 (9), pp.4077-4081. 10.1039/c3cp54484b . hal-01157200

\author{
HAL Id: hal-01157200 \\ https://hal.science/hal-01157200
}

Submitted on 13 Nov 2015

HAL is a multi-disciplinary open access archive for the deposit and dissemination of scientific research documents, whether they are published or not. The documents may come from teaching and research institutions in France or abroad, or from public or private research centers.
L'archive ouverte pluridisciplinaire HAL, est destinée au dépôt et à la diffusion de documents scientifiques de niveau recherche, publiés ou non, émanant des établissements d'enseignement et de recherche français ou étrangers, des laboratoires publics ou privés. 
Cite this: Phys. Chem. Chem. Phys., 2014, 16, 4077

Received 23rd October 2013, Accepted 24th December 2013

DOI: $10.1039 / c 3 c p 54484 b$

www.rsc.org/pccp

\title{
Surface decoration of catanionic vesicles with superparamagnetic iron oxide nanoparticles: a model system for triggered release under moderate temperature conditions $\uparrow$
}

\author{
G. Béalle, ${ }^{a}$ L. Lartigue, ${ }^{b}$ C. Wilhelm, ${ }^{b}$ J. Ravaux, ${ }^{c}$ F. Gazeau, ${ }^{b}$ R. Podor, ${ }^{c}$ D. Carrière*d \\ and C. Ménager*a
}

\begin{abstract}
We report the design of new catanionic vesicles decorated with iron oxide nanoparticles, which could be used as a model system to illustrate controlled delivery of small solutes under mild hyperthermia. Efficient release of fluorescent dye rhodamine 6G was observed when samples were exposed to an oscillating magnetic field. Our system provides direct evidence for reversible permeability upon magnetic stimulation.
\end{abstract}

\section{Introduction}

Magnetic drug carriers made from vesicles, such as polymersomes or liposomes associated with magnetic nanoparticles (MNPs), play a crucial role as vectors for targeting, imaging and drug delivery applications in cancer therapy. In these systems, the MNPs generate heat upon dissipation of an applied oscillating magnetic field, resulting in delivery of the encapsulated solute. In most reports, MNPs are encapsulated in the aqueous core of vectors, and their release occurs upon bulk temperature elevation. ${ }^{1,2}$ However, it has been recently shown that magnetic delivery is also possible with MNPs embedded in the membrane of the vector, be it a liposome or a polymersome. In those studies, solute release occurs without a significant increase in bulk temperature. ${ }^{1,3-7}$ Therefore, it has been clearly shown that a local perturbation is sufficient to trigger delivery. The nature of this perturbation depends on the type of particles used: superparamagnetic iron oxide $\left(\mathrm{Fe}_{2} \mathrm{O}_{3}\right.$ or $\left.\mathrm{Fe}_{3} \mathrm{O}_{4}\right)$ with freely rotating magnetic moments or cobalt ferrite $\left(\mathrm{CoFe}_{2} \mathrm{O}_{4}\right)$ with blocked magnetic moments. Dyes or drugs can thus be released by applying a

\footnotetext{
${ }^{a}$ Laboratoire PECSA-UMR 7195-CNRS, Université Pierre et Marie Curie, UPMC-Univ Paris 06, 4 place Jussieu, 75252 Paris cedex 05, France. E-mail: christine.menager@upmc.fr

${ }^{b}$ Laboratoire Matières et Systèmes Complexes (MSC), UMR 7057 CNRS/Université Paris - Diderot, 10 rue Alice Domon et Léonie Duquet, 75205 Paris cedex 13, France

ICSM, UMR5257 CNRS/CEA/UM2/ENSCM, Site de Marcoule, 30207 Bagnols/Cèze, France

${ }^{d}$ CEA, IRAMIS, UMR 3299 CEA/CNRS, LIONS, F-91191 Gif-sur-Yvette cedex, France. E-mail: david.carriere@cea.fr

$\dagger$ Electronic supplementary information (ESI) available. See DOI: 10.1039/ c3cp54484b
}

high-frequency alternating magnetic field (HF-AMF, $100 \mathrm{kHz}-$ $1 \mathrm{MHz}$ ) or a low-frequency alternating magnetic field (LF-AMF, $\approx \mathrm{kHz}$ ) for the two types of particles respectively. Mechanical perturbations of the membrane occur when cobalt ferrite is used. When iron oxide nanoparticles are used, permeability enhancement of the bilayer is possible thanks to membrane melting or to partial rupture. ${ }^{1,3-6}$ Amstad et al. have clearly established that high frequency magnetic field treatment of liposomes, hosting iron oxide nanoparticles in their membranes, resulted in direct injection of heat into the lipid membrane through local heating of embedded nanoparticles. ${ }^{3}$ This allowed permeability enhancement by increasing the local temperature to the melting point without bulk water heating and without disruption of the vesicles. Instead of embedding MNPs into the bilayer, another approach to induce local heating is to decorate vesicle membranes with MNPs. This strategy has already been used in the case of cobalt ferrite physisorbed on giant unilamellar vesicles subjected to a LF-AMF. ${ }^{8}$ In this paper we show that membrane permeability can also be obtained under HF-AMF. The system designed for this purpose is giant catanionic vesicles with adsorbed superparamagnetic iron oxide nanoparticles.

The model system used for this study is made of a mixture of surfactants containing opposite charges, also known as 'catanionic mixtures'. This type of assembly has already been successfully reported to be suitable for pharmaceutical applications when biocompatible surfactants are used..$^{9-11}$ Here, we focus on the myristic acid-CTAC system which is not biocompatible but shows several fundamental adequate properties for this study. ${ }^{12-14}$ These catanionic vesicles exhibit a transition between solidstate bilayers (so-called "gel phase") and fluid-state bilayers at a well-defined temperature $\left(T_{\mathrm{m}}=57{ }^{\circ} \mathrm{C}\right) ;{ }^{15}$ they show high encapsulation efficiency with retention times in the order of months; 
their size in the micron range allows direct characterization by confocal microscopy of their integrity and permeability; their surface properties are conveniently adjusted by the choice and the respective ratio of the surfactants.

In this paper, we report the preparation of catanionic vesicles based on the myristic acid-CTAC system, with a membrane covered by $\gamma-\mathrm{Fe}_{2} \mathrm{O}_{3}$ superparamagnetic nanoparticles. This paper demonstrates that hydrophilic molecules can be encapsulated, retained and released by applying an oscillating magnetic field without destruction of the vector. This release occurs at moderate bulk temperature, far below the melting point of the bilayer.

\section{Materials and methods}

\section{Magnetic nanoparticle synthesis}

The aqueous suspension of magnetic nanoparticles was prepared by alkaline co-precipitation of $\mathrm{FeCl}_{2}(0.9 \mathrm{~mol})$ and $\mathrm{FeCl}_{3}$ $(1.5 \mathrm{~mol})$ salts, according to Massart's procedure. ${ }^{16}$ Superparamagnetic maghemite grains $\left(\gamma-\mathrm{Fe}_{2} \mathrm{O}_{3}\right)$ were obtained by oxidizing $1.3 \mathrm{~mol}$ of magnetite with $1.3 \mathrm{~mol}$ of iron nitrate (boiling solution). After magnetic decantation, $2 \mathrm{~L}$ of distilled water and $360 \mathrm{~mL}$ of $\mathrm{HNO}_{3} 20 \%$ were added to the solution and the mixture was stirred for $10 \mathrm{~min}$. Prepared maghemite nanoparticles were washed several times with acetone $(3 \times 1 \mathrm{~L})$ and ether $(2 \times 500 \mathrm{~mL})$ and were suspended in water. Size sorting was performed by adding $\mathrm{HNO}_{3}(0.45 \mathrm{M})$ to the suspension followed by magnetic decantation. This operation was repeated with the deposit until a suitable particle size was obtained. Sodium citrate $\left(n_{\mathrm{Fe}} / n_{\mathrm{Cit}}=0.13\right)$ was added to the nanoparticles and the mixture was heated at $80{ }^{\circ} \mathrm{C}$ for $30 \mathrm{~min}$ to promote adsorption of citrate anions onto their surface. Citrated nanoparticles were precipitated in acetone and suspended in water. The volume fraction and average size of the maghemite grains were determined by fitting the magnetization curve of nanoparticles using Langevin's law. Particles of $9 \mathrm{~nm}$ diameter (polydispersity index $\sigma=0.25$, volume fraction of nanoparticles in the suspension $\varphi=7.2 \%, \mathrm{pH}=2$ ) and $7 \mathrm{~nm}(\sigma=0.35, \varphi=2.63 \%, \mathrm{pH}=2)$ were obtained.

\section{Vesicles preparation}

Cetyltrimethylammonium chloride $\left(\mathrm{C}_{16} \mathrm{TA}^{+} \mathrm{Cl}^{-}\right)$and myristic acid $\left(\mathrm{C}_{13} \mathrm{COOH}\right)$ were purchased from Fluka and used as received. The final MNP concentration was adjusted by dilution in $\mathrm{HNO}_{3}$ to $\mathrm{pH}=2$. In a typical $0.33 \%$ wt vesicle preparation, $31.3 \mathrm{mg}$ of cetyltrimethylammonium chloride, $41.8 \mathrm{mg}$ of myristic acid and $7.497 \mathrm{~g}$ of MNPs suspension, and optionally $693 \mu \mathrm{L}$ of rhodamine 6G $100 \mu \mathrm{M}$ were stirred at $50{ }^{\circ} \mathrm{C}$ for 3 days. The solution is then diluted 3-fold with water and stirred at $50{ }^{\circ} \mathrm{C}$ for 30 minutes and dialyzed (Float-a-Lyzer, 8-10 kDa, Sigma) at room temperature against $500 \mathrm{~mL}$ of water (MilliQ). Dialysis baths were changed every hour for the first 8 hours, and then every 12 hours for the next 4 days. To eliminate excess of aggregated nanoparticles, $1 \mathrm{~mL}$ of the sample was diluted 3-fold in water (MilliQ) and centrifuged for $20 \mathrm{~min}$ at $201 \mathrm{~g}$. For surface-decorated vesicles, the supernatant was recovered while the sediment was collected for surfacedecorated vesicles encapsulating MNPs.

\section{Characterization}

Confocal microscopy. Vesicles were examined using a confocal laser scanning inverse microscope (Olympus IX 81) equipped with a $\times 60$ magnification/1.42 NA oil objective lens coupled to an FV 1000 confocal head (Olympus). Rhodamine 6G (encapsulated or added) was excited at $543 \mathrm{~nm}$ and the emission collected above $560 \mathrm{~nm}$.

Transmission electron microscopy (TEM). Magnetic vesicles were diluted after centrifugation and single droplets were deposited on a carbon coated copper grid for drying. Observations were performed using a JEOL $100 \mathrm{CX}$ Transmission Electronic at $60 \mathrm{keV}$.

Magnetophoresis experiments. A home-made magnetophoresis setup previously described was used. ${ }^{17}$ Magnetophoretic mobilities of individual vesicles were measured in a $145 \mathrm{mT}$ magnetic field with a magnetic field gradient of $195 \mathrm{~T} \mathrm{~m}^{-1}$ created by a magnetized nickel rod (50 $\mu \mathrm{m}$ in diameter).

Wet scanning transmission electron microscopy (Wet-STEM). A FEI Quanta 200 ESEM FEG microscope operated at $30 \mathrm{keV}$ was used, the nominal resolution being $1 \mathrm{~nm}$. A Peltier stage and an inverse BSE detector are located below the final lens in the microscope chamber. A $5 \mu$ d droplet of the diluted sample solution was deposited on a TEM copper grid coated with a holey carbon film. The grid was then placed on the Peltier stage previously cooled at $T=3{ }^{\circ} \mathrm{C}$. An optimized pump down sequence was used to adjust the water partial pressure at $1001 \mathrm{~Pa}$. Afterwards, the pressure is adjusted to evaporate a very small amount of water from the droplet to adjust the water film thickness and allow sample observation.

Hyperthermia experiments. A laboratory-made device was used. ${ }^{18}$ The system is a resonant RLC circuit producing a magnetic field (frequency $=520 \mathrm{kHz}$, amplitude $=28 \mathrm{kA} \mathrm{m}^{-1}$ ) in a $16 \mathrm{~mm}$ coil. The sample was heated at $37^{\circ} \mathrm{C}$ before the application of the oscillating magnetic field, and the temperature was probed using a fluorooptic fiber thermometer.

\section{Results and discussion}

\section{Control of the vesicle structure}

The preparation of magnetically decorated vesicles relies on a fine control of the nanoparticle-vesicle interactions during the preparation, repulsive during vesicle formation, and attractive after vesicle formation.

The magnetic vesicles are prepared as follows: myristic acid and cetyltrimethylammonium chloride are co-solubilized in a solution of positively charged maghemite nanoparticles (either 7 or $9 \mathrm{~nm}$, TEM characterization available in Fig. S1, ESI $\dagger$ ) in acidic media $\left(\mathrm{HNO}_{3}, \mathrm{pH}=2\right)$. As a result, surfactants self-assemble to form hollow elongated vesicles and release additional $\mathrm{HCl} 2 \mathrm{mM}$ upon association (Fig. 1, step a). ${ }^{13}$ At this step of the preparation, the vesicles and the magnetic nanoparticles (isoelectric point at $\mathrm{pH}=7$, Fig. S2, ESI $\dagger$ ) bear a positive surface charge. ${ }^{19}$ The vesicle-nanoparticle mixture is then dialyzed against MilliQ water, which allows both (i) elimination of the ions present in the bulk while $\mathrm{HCl} / \mathrm{HNO}_{3}$ remains trapped in the core, resulting in an osmotic swelling of the elongated vesicles into spherical 


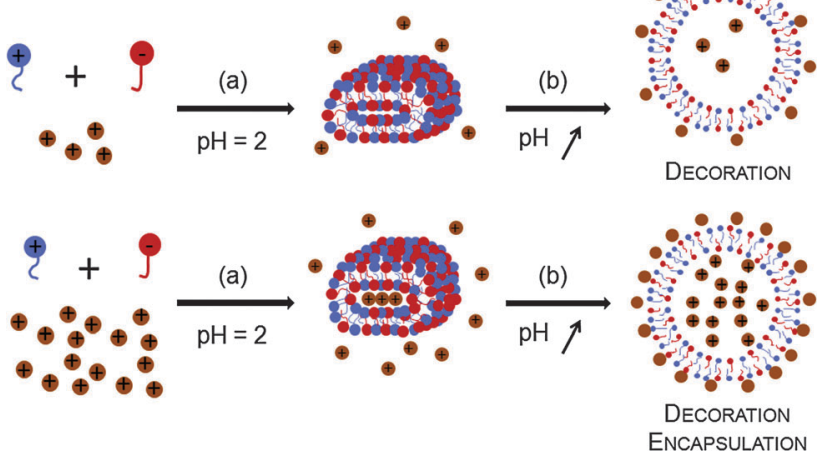

Fig. 1 Sketch of the preparation of catanionic vesicles with MNPs adsorbed on the surface (upper line) and both adsorbed on the surface and encapsulated into the core of the vesicles (lower line). Step (a): stirring process at $50{ }^{\circ} \mathrm{C}$ for 3 days. Surfactants co-solubilize and form hollow elongated vesicles. Step (b): dialysis against water during 5 days.

vesicles (Fig. 1, step b); ${ }^{12,13}$ and (ii) an increase of the $\mathrm{pH}$ of the solution until the charge of the nanoparticles is sufficiently reduced, leading to their precipitation onto the surface of the vesicles.

Concentration of MNPs influenced the vesicles structure, leading either to decorated vesicles or decorated vesicles encapsulating MNPs as illustrated in Fig. 1. When the preparation is performed with a suspension of MNPs at $0.16 \mathrm{M}$ in iron, decorated vesicles are obtained. Standard optical microscopy transmission mode reveals that the surface coverage of dialyzed vesicles by magnetic nanoparticles results in an enhancement of membrane contrast (Fig. S3, ESI $\dagger$ ). This is usually difficult to observe in pristine vesicle samples. We assume that a slight encapsulation occurs in the core but MNPs are clearly not numerous enough to be observed. Further investigations with TEM revealed that groups of catanionic vesicles as well as isolated ones are clearly visible thanks to nanoparticles adsorption onto the surface in the form of independent MNPs or small clusters (Fig. 2a). The entire solution remains stable (no flocculation) if the quantity

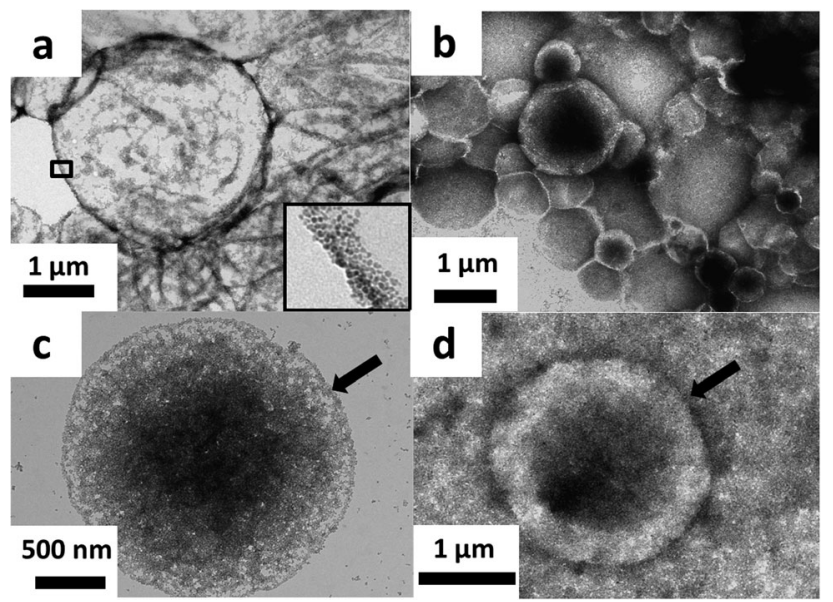

Fig. 2 Vesicles decorated with $7 \mathrm{~nm}$ MNPs, observed by TEM (a). Vesicles with $9 \mathrm{~nm}$ MNPs adsorbed on the surface and encapsulated, observed by TEM (b) and supplemented with high magnification (c, d).
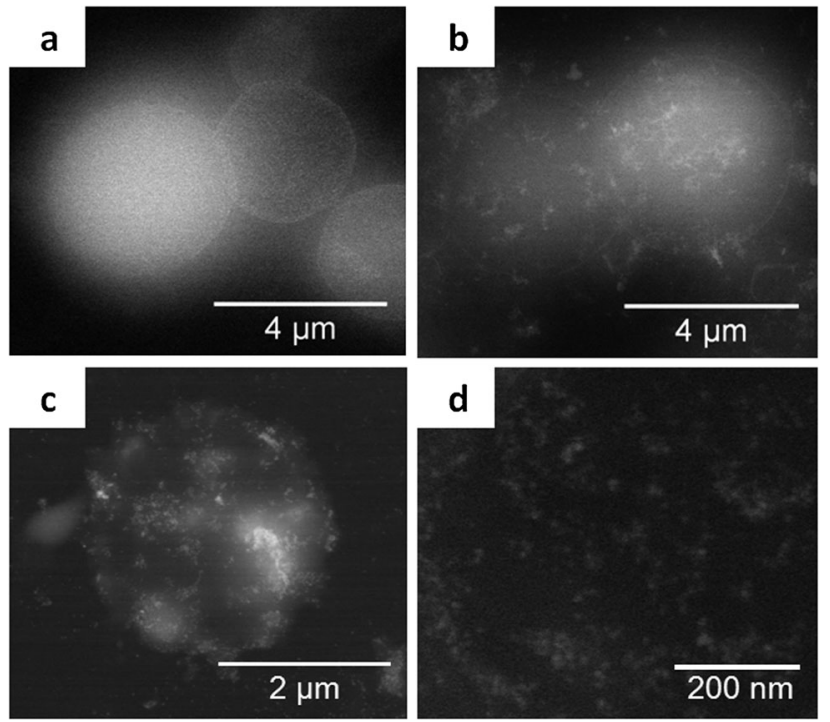

Fig. 3 Wet-STEM images of control vesicles (a), $7 \mathrm{~nm}$ magnetic nanoparticles adsorbed on vesicles $(b, c)$ and $7 \mathrm{~nm}$ magnetic nanoparticles in solution (d), examined by wet-STEM at $30 \mathrm{keV}$.

of nanoparticles introduced during vesicles preparation is not sufficient to cover the whole surface of vesicles. The latter being slightly positively charged at neutral $\mathrm{pH}$, the stability of the solution is maintained.

Because drying artifacts can influence the distribution of MNPs on the vesicles, we confirmed our results using a recently developed transmission mode associated with the Environmental Scanning Electron Microscope called "Wet-STEM" (Wet-scanning TEM). ${ }^{20-22}$ A low water pressure is applied around the sample enabling direct imaging of insulating, wet samples in the native state. The wet-STEM imaging mode is perfectly adapted to the direct observation of nanoparticles and materials such as colloids, vesicles or cells in water media. ${ }^{21,23,24}$ Vesicles of the control sample observed in water show homogeneous contrast (Fig. 3a), whereas the MNPs, seen as white dots (Fig. 3d), are distinctly covering the vesicles as small clusters (Fig. $3 \mathrm{~b}$ and $\mathrm{c}$ ). This unambiguously confirms the surface covering of vesicles by MNPs. In addition, it is observed that the MNPs are agglomerated into surface clusters, and that the membrane is not fully covered by the nanoparticles.

When the concentration of MNPs introduced during preparation is increased, the surface of the vesicles finally gets saturated. At a nominal iron concentration of $2.2 \mathrm{M}$, the vesicles encapsulate MNPs in their cores, as evidenced in optical transmission mode by the typical vesicles/solution contrast (results not shown). In the cores, the nanoparticles do not seem to be aggregated, which is assigned to the encapsulation of $\mathrm{HCl}$ (from vesicle formation) and $\mathrm{HNO}_{3}$ (from the MNPs solution), which maintain the $\mathrm{pH}$ below the isoelectric point. This confirms that encapsulation of both MNPs and small ionic species occurs during the co-solubilization and formation of impermeable elongated vesicles (Fig. 1, step a). Loaded vesicles also appeared covered by maghemite grains, as demonstrated by TEM pictures (Fig. 2b-d).

This suggests that a quantified amount of nanoparticles can be adsorbed on the surface until saturation is reached, and the 
excess MNPs are encapsulated. The ideal nanoparticle concentration required for full surface coverage saturation can be estimated by comparing the available vesicle surface and the projected area of nanoparticles per solution volume unit (Appendix S1, ESI $\dagger$ ). For a surfactant concentration of $0.33 \%$ (wt/wt), an average vesicle diameter of $5 \mu \mathrm{m}$ and $9 \mathrm{~nm}$ nanoparticles, the approximate concentration of iron would be $1.9 \mathrm{M}$ (Appendix S1, ESI $\dagger$ ), which suits very well with experimental observations. Due to complete neutralization of the surface by nanoparticles, the stability of vesicles in water is slightly decreased and objects tend to settle.

\section{Determination of the magnetic load}

Both systems (with surface decoration only and with surface decoration and encapsulation of MNPs) were characterized for their magnetophoretic mobilities in order to determine the quantity of MNPs on/in the vesicles. The home-made chamber used for the experiment consists of two superposed glass slides with a nickel rod placed between two slides. ${ }^{17}$ The magnetization of the nickel rod creates a strong magnetic field gradient enabling the vesicles to migrate and accumulate onto the rod (Fig. S4, ESI $\dagger$ ). The observed magnetic mobilities in the $195 \mathrm{~T} \mathrm{~m}^{-1}$ magnetic gradient are $51 \pm 17 \mu \mathrm{m} \mathrm{s}^{-1}$ and $115 \pm 46 \mu \mathrm{m} \mathrm{s}^{-1}$ for vesicles with MNPs absorbed on the surface and vesicles with MNPs both absorbed/encapsulated on/in vesicles, respectively. These data lead to an iron content of $0.18 \pm 0.11 \mathrm{pg}$ per vesicle and $0.43 \pm 0.26 \mathrm{pg}$ per vesicle, respectively. Not surprisingly, vesicles with MNPs both absorbed and encapsulated show the best magnetic mobility and the highest iron content per vesicle. However, the magnetophoresis experiments also underline that vesicles with surface decoration show relevant migration under the magnetic field despite the lower MNPs content.

More quantitatively, the iron content per vesicle corresponds to $1.2 \times 10^{5}$ MNPs for decorated vesicles which can be expressed as a covering surface of $36.3 \mu \mathrm{m}^{2}$ for charged nanoparticles. For decorated vesicles encapsulating nanoparticles, the iron content per vesicle corresponds to $2.9 \times 10^{5}$ MNPs or a covering surface of $93.8 \mu \mathrm{m}^{2}$. These data are in reasonable agreement with that expected for full surface coverage of a $5 \mu \mathrm{m}$ vesicle $\left(78.5 \mu \mathrm{m}^{2}\right)$. Decorated vesicles are thus $50 \%$ covered by nanoparticles. For decorated vesicles encapsulating MNPs, the covering surface is higher than full coverage, which confirms that vesicles are $100 \%$ covered by MNPs and encapsulate them at the same time. This outlines that almost all MNPs present in solution cover the vesicles, and therefore suggests that a strong attraction between particles and the catanionic vesicles takes over once the $\mathrm{pH}$ is permuted. The exact nature of this interaction needs to be elucidated, but at this stage we still may invoke a specific interaction between the $\gamma-\mathrm{Fe}_{2} \mathrm{O}_{3}$ particles and the carboxylic headgroups of the vesicles, or strong dipolar interactions between the residual charge of the particles and the mixed $+/-$ surface. $^{25}$

\section{Release upon oscillating magnetic field stimulation}

These surface decorated vesicles are of special interest for hyperthermia because as previous studies report a local heating at the membrane level is possible which allows the release of encapsulated
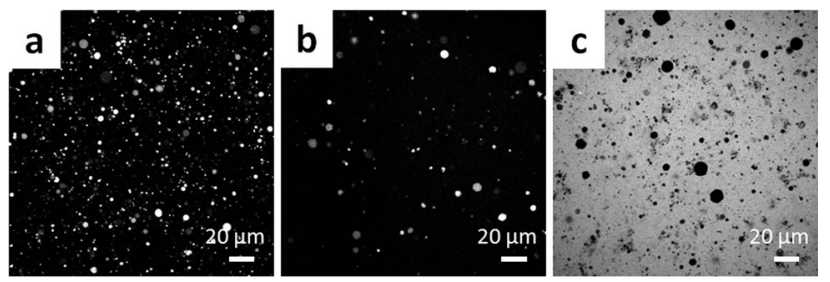

Fig. 4 Vesicles covered with $9 \mathrm{~nm}$ MNPs observed in the confocal mode before (a) and after (b) hyperthermia experiments. Addition of rhodamine 6G in the bulk (c) demonstrates that vesicles are still intact and impermeable after the experiment.

species. A cationic water-soluble dye (rhodamine $6 \mathrm{G}, \lambda_{\text {exc }}=543 \mathrm{~nm}$ ) can be encapsulated into the vesicles with magnetic surface coverage via addition during the initial surfactant co-solubilization. During the subsequent dialysis step, the excess dye is removed from the exterior of the vesicles, leading to bright vesicles in confocal laser microscopy (Fig. 4a). After stimulation by an oscillating magnetic field $\left(28 \mathrm{kA} \mathrm{m}^{-1}, 520 \mathrm{kHz}\right)$ for 30 minutes, we observe that the vesicles release their content (Fig. 4b). ${ }^{18}$ Addition of an aliquot of rhodamine to the stimulated solution revealed that a significant fraction of vesicles remained intact (Fig. 4c) and are impermeable once the stimulation had stopped. Very strikingly, this release of the encapsulated solute occurs with a mild temperature increase of the solution during stimulation $\left(T_{0}=\right.$ $37.6{ }^{\circ} \mathrm{C}, T_{\max }=42.6{ }^{\circ} \mathrm{C}$, Fig. S5, Table S1, ESI $\dagger$ ). This demonstrates the interest in magnetic surface decoration brought about by our system: the MNPs are efficiently distributed, i.e. they are directly in contact with the bilayer, which allows release of the solutes with a minimum amount of heat generation. More quantitatively, by counting the number of decorated vesicles before and after magnetic stimulation (based on 1100 vesicles), we can estimate that $81 \% \pm 2 \%$ of the vesicles released the dye. After mild hyperthermia, $73 \% \pm 4 \%$ of the vesicles were impermeable again and $27 \%$ of the vesicles were destroyed during the stimulation. Similar results were obtained for decorated vesicles encapsulating MNPs.

As in the case of other magnetic bilayers, this system allows release of the solutes with a minimum heat generation., ${ }^{3,6}$ The exact mechanism of release is difficult to identify, but we may assign it to a permeability enhancement of the bilayer by melting. At room temperature, catanionic vesicles are 'frozen', i.e. the bilayer is in the solid state, as demonstrated by previous WAXS (Wide Angle X-ray Scattering) experiments. ${ }^{19}$ This quenched state contrasts with usual catanionic vesicles which are in thermodynamic equilibrium, and accounts for their stability against ageing or dilution, or their low permeability to ions or small solutes. As a consequence, rhodamine remains trapped in magnetic vesicles for months. However, we outline that the bulk solution temperature always remains below the usual melting point of these catanionic vesicles $\left(T_{\mathrm{m}}=\right.$ $57{ }^{\circ} \mathrm{C}, \Delta H=18 \mathrm{~kJ} \mathrm{~mol}^{-1}$ of the surfactant chain) demonstrating that the temperature increase could be localized in the bilayer. ${ }^{15}$

\section{Conclusions}

In summary, we demonstrate the preparation of magnetic gelphase catanionic vesicles decorated with superparamagnetic 
nanoparticles, which enable on-demand release of encapsulated solutes. Remarkably, the magnetic delivery occurs with a low temperature increase of the bulk, theoretically not sufficient to induce the gel-liquid phase transition and thus the permeability enhancement of the membrane. A local temperature increase around the membrane could explain the release of the dye, as observed by other groups. Our results complete previous studies ${ }^{3,6}$ by providing direct evidence of the reversibility of bilayer permeation upon magnetic stimulation. Current investigations will further exploit our model systems by performing in situ, time-resolved measurements of permeability on single vesicles.

The possibility to formulate similar vectors with biocompatible surfactants, combined with their versatile functions, make these vesicles a promising alternative to poorly selective vectors. ${ }^{11}$ Vesicles such as these may be used in the future for targeting, imaging and on-demand delivery of pharmaceutical drugs, cosmetics or cells and bacteria in vitro or in vivo.

\section{Acknowledgements}

We kindly acknowledge Delphine Talbot for the preparation and characterization of magnetic nanoparticles. We also thank Sophie Neveu and Aude Michel for the TEM pictures.

\section{Notes and references}

1 S. Nappini, M. Bonini, F. B. Bombelli, F. Pineider, C. L. Sangregorio, P. Baglioni and B. Norden, Soft Matter, 2011, 7, 1025-1037.

2 L. A. Tai, P. J. Tsai, Y. C. Wang, Y. J. Wang, L. W. Lo and C. S. Yang, Nanotechnology, 2009, 20, 135101.

3 E. Amstad, J. Kohlbrecher, E. Muller, T. Schweizer, M. Textor and E. Reimhult, Nano Lett., 2011, 11, 1664-1670. 4 S. Nappini, M. Bonini, F. Ridi and P. Baglioni, Soft Matter, 2011, 7, 4801-4811.

5 Y. Chen, A. Bose and G. D. Bothun, ACS Nano, 2010, 4, 3215-3221.

6 C. Sanson, O. Diou, J. Thevenot, E. Ibarboure, A. Soum, A. Brulet, S. Miraux, E. Thiaudiere, S. Tan, A. Brisson, V. Dupuis, O. Sandre and S. Lecommandoux, ACS Nano, 2011, 5, 1122-1140.
7 X. Ding, K. Cai, Z. Luo, J. Li, Y. Hu and X. Shen, Nanoscale, 2012, 4, 6289-6292.

8 S. Nappini, T. Al Kayal, D. Berti, B. Nordèn and P. Baglioni, J. Phys. Chem. Lett., 2011, 2, 713-718.

9 E. W. Kaler, A. K. Murthy, B. E. Rodriguez and J. A. Zasadzinski, Science, 1989, 245, 1371-1374.

10 A. Boudier, P. Castagnos, E. Soussan, G. Beaune, H. Belkhelfa, C. Ménager, V. Cabuil, L. Haddioui, C. Roques, I. Rico-Lattes and M. Blanzat, Int. J. Pharm., 2011, 403, 230-236.

11 S. Consola, M. Blanzat, E. Perez, J. C. Garrigues, P. Bordat and I. Rico-Lattes, Chem.-Eur. J., 2007, 13, 3039-3047.

12 Y. Michina, D. Carrière, C. Mariet, M. Moskura, P. Berthault, L. Belloni and T. Zemb, Langmuir, 2009, 25, 698-706.

13 D. Kopetzki, Y. Michina, T. Gustavsson and D. Carrière, Soft Matter, 2009, 5, 4212-4218.

14 G. Béalle, J. Jestin and D. Carrière, Soft Matter, 2011, 7, 1084-1089.

15 Y. Michina, D. Carrière, T. Charpentier, R. Brito, E. F. Marques, J. P. Douliez and T. Zemb, J. Phys. Chem. B, 2010, 114, 1932-1938.

16 R. Massart, IEEE Trans. Magn., 1981, 17, 1247.

17 C. Wilhelm, F. Lavialle, C. Péchoux, I. Tatischeff and F. Gazeau, Small, 2008, 4, 577.

18 J. P. Fortin, C. Wilhelm, J. Servais, C. Menager, J. C. Bacri and F. Gazeau, J. Am. Chem. Soc., 2007, 129, 2628-2635.

19 E. Maurer, L. Belloni, T. Zemb and D. Carrière, Langmuir, 2007, 23, 6554-6560.

20 D. Stokes, Principles and practice of variable pressure: environmental scanning electron microscopy (VP-ESEM), The Atrium, Southern Gate, Chichster, West Sussex, UK, 2008.

21 A. Bogner, G. Thollet, D. Basset, P. H. Jouneau and C. Gauthier, Ultramicroscopy, 2005, 104, 290-301.

22 A. M. Donald, Nat. Mater., 2003, 2, 511-516.

23 V. A. Maraloiu, M. Hamoudeh, H. Fessi and M. G. Blanchin, J. Colloid Interface Sci., 2010, 352, 386-392.

24 N. de Jonge, D. B. Peckys, G. J. Kremersa and D. W. Piston, Proc. Natl. Acad. Sci. U. S. A., 2009, 106, 2159-2164.

25 D. Carrière, L. Belloni, B. Demé, M. Dubois, C. Vautrin, A. Meister and T. Zemb, Soft Matter, 2009, 5, 4983. 